$35-40$

\title{
The effect of body mass index on lumbar lordosis on the Mizuho OSI Jackson spinal table
}

\author{
Authors Justin Bundy, Tommy Hernandez, Haitao Zhou, Norman Chutkan \\ Institution Orthopaedic Department, Medical College of Georgia, Augusta, Georgia, USA
}

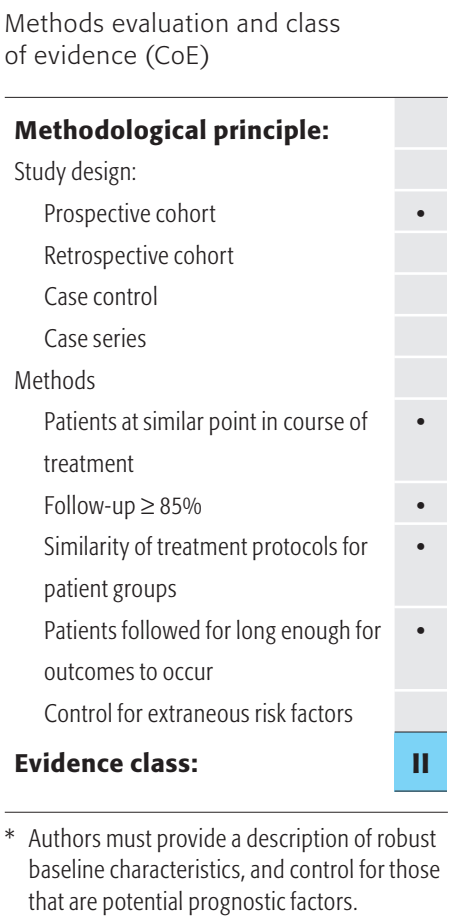

The definiton of the different classes of evidence is available on page 83.

\section{ABSTRACT}

Study design: Prospective cohort study.

Clinical question: Does the patients' body mass index (BMI) influence the degree of intraoperative lumbar lordosis in patients undergoing operative treatment on the Mizuho Orthopedic Systems Incorporated (OSI) Jackson spinal table?

Methods: Twenty-four consecutive patients undergoing posterior spinal instrumentation and fusion on the Jackson table, excluding those with sagittal malalignment, underwent standing preoperative and prone intraoperative lateral x-rays. Intervertebral body angle measurements were obtained from Ll-Sl using the modified method of Cobb. Changes in angle measurements were compared to BMI using linear regression and ANOVA.

Results: We found a mean lordosis of $52.6^{\circ}$ in standing preoperative x-rays compared to a prone position mean lordosis of $61.5^{\circ}$ on the Jackson table. The mean change was $8.88^{\circ}$ with a range of $0^{\circ}-18^{\circ}$. A linear association between lordosis and BMI was demonstrated $(P<.0022)$. As BMI increased, so did lordosis (correlation coefficient, 0.59).

Conclusions: The current study is the first in which a correlation of patient body mass and use of the Jackson table has been evaluated. These data suggest that BMI influences lumbar lordosis on the Jackson table and that care must be used when dealing with a population with large BMI on the Jackson table. 


\section{STUDY RATIONALE AND CONTEXT}

Prone patient position can influence lumbar spine surgical techniques and reconstruction results due to changes in spinal alignment. Studies have investigated patient positioning, especially in regard to various operative frames and their effect on sagittal alignment. In general, mechanical decompression of the abdomen is desirable for spinal procedures carried out in a prone position in order to decompress the epigastric plexus and hopefully thus diminish epidural bleeding. Prolonged prone position without external mechanical decompression of the lower torso could also lead to damage to internal organs. These concerns are amplified in an overweight patient population. Unfortunately, there is an absence of information regarding patient body habitus as it relates to intraoperative alignment of spinal surgery done in a prone position.

\section{CLINICAL QUESTION}

Does a patient's body mass index (BMI) affect lumbar lordosis of patients undergoing lumbosacral posterior fusion surgery in a prone position on a commonly used spinal table (Mizuho Orthopedic Systems Incorporated (OSI) Jackson table)?

\section{METHODS}

Study design: Prospective cohort study.

Inclusion criteria (Fig 1): From July 2005 to December 2005, all patients who underwent instrumented posterior lumbosacral fusion by the corresponding author were included in the study.

Exclusion criteria: Patients with preoperatively present sagittal or coronal plane deformities greater than $10^{\circ}$ in either coronal or sagittal direction diagnosed in the preoperative workup were excluded. Patients with previous lumbar fusion or spondylolisthesis were excluded.

Outcomes and prognostic (risk) factors to be evaluated:

- During preoperative evaluation, all patients were weighed on a single digital scale and measured using a wall tape by the same clinical nurse. BMI was determined by taking weight over the square of height $(\mathrm{kg} / \mathrm{m} 2)$ [1].

- On the preoperative standing lateral and intraoperative prone lateral $\mathrm{x}$-rays, lumbar lordosis measurements were performed from Ll-Sl using the modified method of Cobb with images centered on the vertebral body of L3 (Fig 2a-b).

- Two examiners performed measurements. All measurements were completed in each patient by one examiner to maintain consistency.

Analysis:

- Intraobserver measurement error was evaluated and found to be less than $3^{\circ}$.

- Statistical analysis was performed using the paired t-test, ANOVA and linear regression.

Additional information is available in the web appendix at www.aospine.org/ebsj.

\section{RESULTS}

- The mean BMI in this population was $32.5( \pm 4.4)$ and $88 \%$ of patients were considered overweight or obese (Table 1).

- The mean lumbar lordosis angle from Ll to the sacrum with subjects in a standing position was $52.6^{\circ}$ $\left(35^{\circ}-75^{\circ}\right)$ The mean lumbar lordosis on the Jackson table was $61.5^{\circ}\left(38^{\circ}-80^{\circ}\right)$ (Table 2). 


\section{Fig 1 Patient sampling and selection}

\section{Total patients receiving posterior} lumbosacral fusion $(n=32)$

Sagittal or coronal plane deformity greater than $10^{\circ}(n=8)$

Patients available for analysis $(n=24)$
Table 1 Patient BMI distribution in this population

\begin{tabular}{lc} 
Adult BMI Category [1] & n (\%) \\
\hline Underweight $(<18.5)$ & 0 \\
\hline Normal (18.5-24.9) & $2(8.3)$ \\
\hline Overweight $(25.0-24.9)$ & $3(12.5)$ \\
\hline Obese $(\geq 30)$ & $19(79.2)$ \\
\hline
\end{tabular}

Fig 2a-b

a Standing preoperative lateral lumbar spine film b Intraoperative fluoroscopic film in the prone position showing an increase in lumbar lordosis of $5^{\circ}$

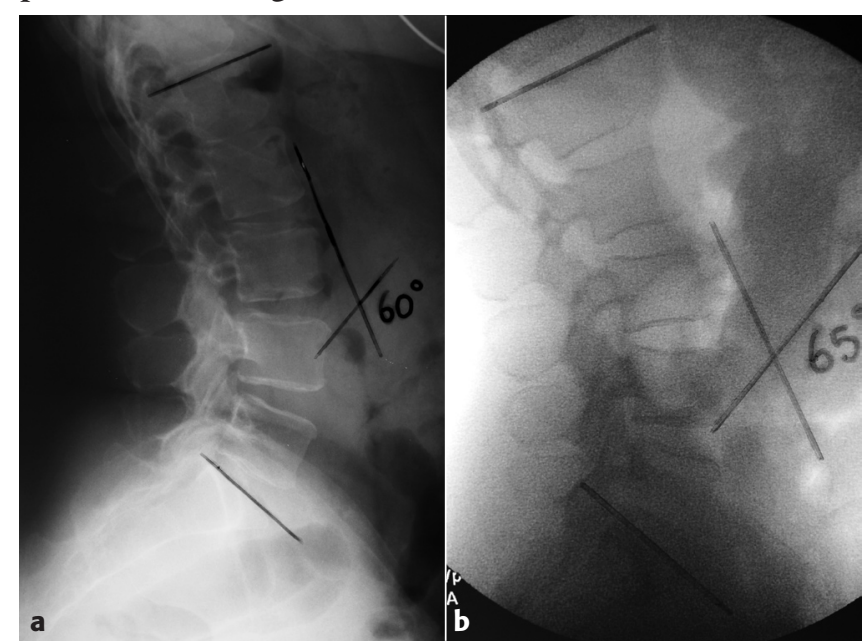

Table 2 Standing and prone lumbar lordosis, change of lordosis and BMI of each patients

\begin{tabular}{|c|c|c|c|c|}
\hline Patient & $\begin{array}{l}\text { Standing } \\
\text { lordosis }\end{array}$ & $\begin{array}{l}\text { Prone } \\
\text { lordosis }\end{array}$ & $\begin{array}{l}\text { Change in } \\
\text { lordosis }\end{array}$ & BMI \\
\hline 1 & 58 & 62 & 4 & 28 \\
\hline 2 & 60 & 68 & 8 & 34 \\
\hline 3 & 75 & 80 & 5 & 32 \\
\hline 4 & 50 & 60 & 10 & 32 \\
\hline 5 & 60 & 76 & 16 & 36 \\
\hline 6 & 41 & 41 & 0 & 30 \\
\hline 7 & 51 & 53 & 2 & 32 \\
\hline 8 & 50 & 58 & 8 & 22 \\
\hline 9 & 45 & 58 & 13 & 46 \\
\hline 10 & 47 & 50 & 3 & 30 \\
\hline 11 & 44 & 56 & 12 & 31 \\
\hline 12 & 50 & 52 & 2 & 30 \\
\hline 13 & 35 & 38 & 3 & 30 \\
\hline 14 & 47 & 65 & 18 & 34 \\
\hline 15 & 60 & 75 & 15 & 37 \\
\hline 16 & 58 & 74 & 16 & 34 \\
\hline 17 & 60 & 77 & 17 & 49 \\
\hline 18 & 67 & 69 & 2 & 25 \\
\hline 19 & 54 & 68 & 14 & 42 \\
\hline 20 & 54 & 68 & 14 & 29 \\
\hline 21 & 56 & 60 & 4 & 31 \\
\hline 22 & 42 & 52 & 10 & 29 \\
\hline 23 & 50 & 62 & 12 & 34 \\
\hline 24 & 50 & 55 & 5 & 24 \\
\hline Mean ( \pm sd) & $52.6( \pm 6.9)$ & $61.5( \pm 8.8)$ & $8.9( \pm 5.0)$ & $32.5( \pm 4.4)$ \\
\hline
\end{tabular}

$\mathrm{sd}=$ standard deviation 
- The increase in lordosis was statistically significant. Measurements of total lordosis preoperatively and postoperatively by the same observer were very reproducible and not significantly different.

- A linear association between increasing BMI and increasing lordosis was seen $(P=.00215)$. An R-squared value of 0.345 suggests that $35 \%$ of the change in angle noted may be due to BMI (Fig 3).

Fig 3 Scatter plot of BMI measurements and change in lordosis from standing to prone position, derived from linear regression, showing strong correlation between change and increasing BMI

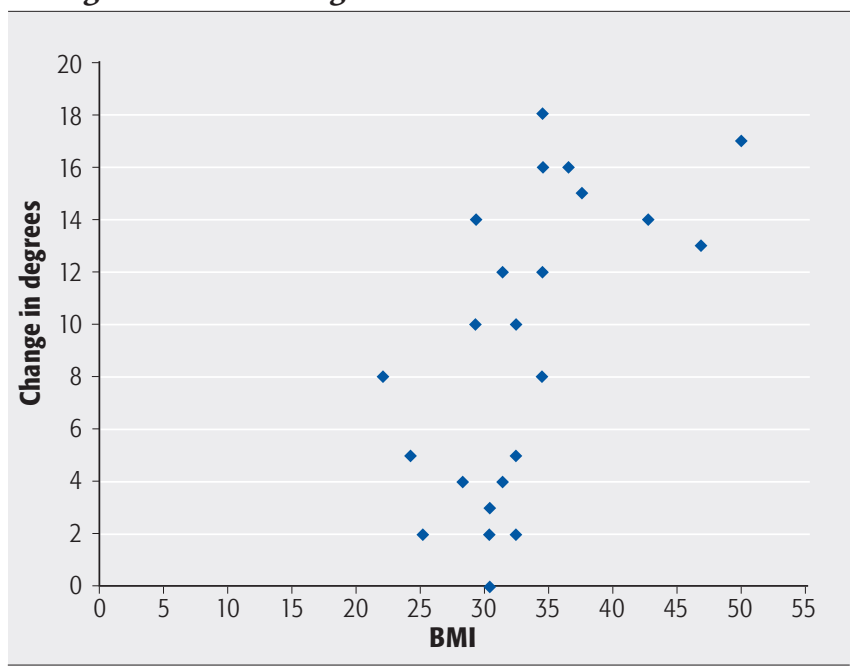

\section{DISCUSSION}

- In lumbar spine posterior fusion surgery it is desirable to maintain or achieve physiologic lordosis. This effort can be helped by thoughtful intraoperative positioning and patient selection. Inadequate restoration of sagittal balance has been implicated as a factor in post fusion surgery low back pain [2, 3].

- Long-term studies have shown that excessive kyphosis of the lumbar spine causes a flat back syndrome and compensatory hyperlordosis below the levels may predispose patients to accelerated degenerative changes [4-6].

- Conversely, fusion in lumbar hyperlordois can lead to increased compensatory malalignment of other levels.

- A statistically significant increase in lumbar lordosis in patients with increasing BMI was seen. It appears that this is caused by the combination of increased patient trunk weight and the table configuration, which leaves the prone patient's lower torso half suspended. To our knowledge this finding has not been reported before.

- Care must be taken when selecting overweight or obese patients for positioning on a Jackson spinal table due to its effects on increasing lumbar lordosis. Efforts to restore physiologic sagittal balance of the spinal column can include preoperative repositioning of patients and intraoperative corrective measures.

- Clinical correlations of iatrogenically induced hyperlordosis and its longterm sequela in overweight patients using this positioning technique are not yet fully understood.

- As result of our study we have heightened our awareness of lumbar alignment changes in overweight patients receiving spinal fusion surgery in a prone position. We have expanded our study focus to include a variety of spinal table alternatives and patient weight categories (Figs $\mathbf{4 , 5}$ ).

\section{SUMMARY AND CONCLUSIONS: KEY POINTS}

- Overweight or obese patients have a significant radiographic and possibly clinical lordotic change of alignment when placed prone on a Jackson table.

- The increase in lumbar lordosis on the Jackson table with increasing BMI noted in our study should be considered by an operating surgeon during posterior lumbar fusion surgery as it might affect postoperative lumbar alignment and clinical outcome. 


\section{REFERENCES}

1. Centers for Disease Control and Prevention: Health weight. It's not a diet, it's a lifestyle. http://www.cdc. gov/healthyweight/assessing/bmi/adult_bmi/; last accessed March 11, 2010.

2. Cochran T, Irstam L, Nachesmson A (1993) Long term anatomic and functional changes in patients with adolescent idiopathic scoliosis treated by Harrington rod fusion. Spine; 8: 577-584.

3. Hayes M, Tomkins S, Herndon W, et al (1988) Clinical and radiographic evaluation of lumbosacral motion below fusion levels in idiopathic scoliosis. Spine; 13:1161-1167.
Fig 4 Positioning overweight patients for prone spine surgery is a well known and, as of yet, incompletely resolved challenge. While the primary concerns often center around cardiopulmonary function for patients with large body mass, decompression of the epigastric plexus by avoiding any direct pressure of the abdominal contents is a major concern as well. The Mizuho OSI Jackson spinal table offers excellent abdominal organ decompression as depicted, but may accentuate lumbar lordosis by anterior pull on the lumbar spine created by a large panniculus.

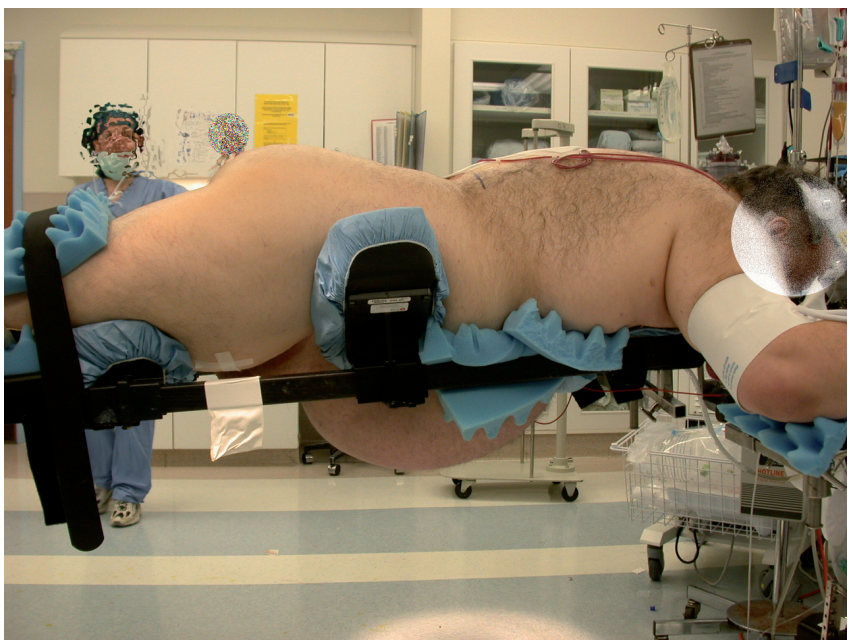

4. Davies AG, McMaster MJ (1992) The effect of Luque rod instrumentation on the sagittal contour of the lumbosacral spine in adolescent idiopathic scoliosis and the preservation of a physiologic lumbar lordosis. Spine; 17: 112-115.

5. LaGrone MO (1988) Loss of lumbar lordosis: Complications of spinal fusion for scoliosis. Orthop Clin North Am; 19: 383-393.

6. Phillips WA, DeWald RL (1985) A comparison of Luque segmental instrumentation with Harrington rod instrumentation in the management of idiopathic scoliosis. Orthop Trans; 9:437-438.
Fig 5 An alternative to a Jackson table is the Mizuho OSI Wilson frame which bends the trunk forward to ease surgical exposure. Depicted here is a Wilson frame which affords free suspension of the abdominal structures, however is limited by the width of the aperture, which may be too narrow, as well as height. Difficulties may also arise while positioning large patients with short necks due to inability to achieve a neutral neck position. Ultimately the choice of spinal table in large patients is a compromise between spinal alignment needs and patient safety.

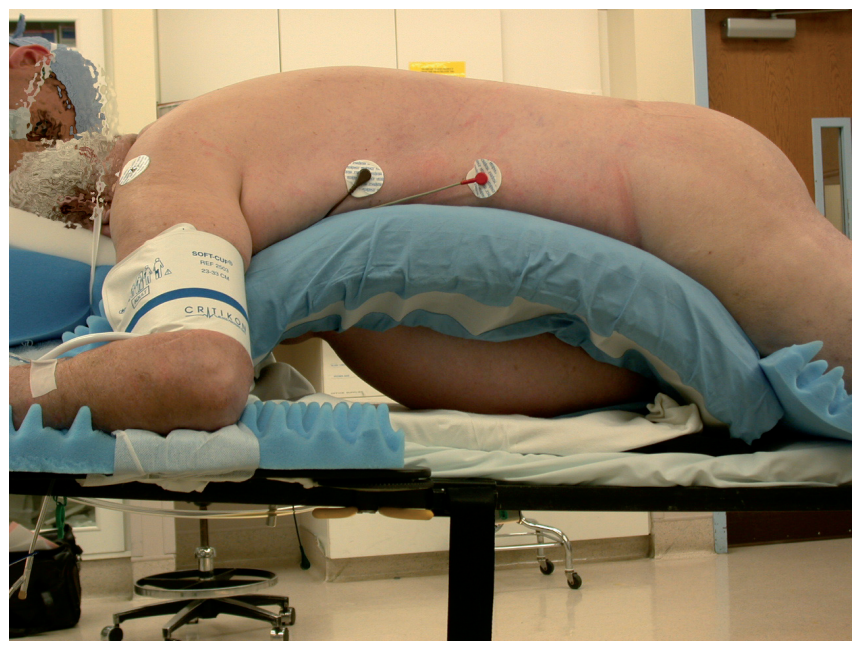




\section{EDITORIAL STAFF PERSPECTIVES}

This is a CoE II prognostic study.

This is a novel study which provides a valuable perspective on the need to consider patient body habitus and its potential impact on maintaining appropriate lordosis. Certainly the finding that lumbar lordosis disproportionally increases in patients with higher BMI's when positioned prone on a Jackson spinal table, which leaves the abdomen freely suspended, is noteworthy for intraoperative consideration. To further evaluate this phenomenon and provide context for these findings, a few methodological points deserve consideration:

What is a "strong" correlation? A correlation of 0.59 may not be considered a "strong" correlation. The sample size is small and addition of a correlation line to the scatter plot would confirm that there is a lot of variation around it. Particularly in a cohort where potentially confounding factors (eg, age, sex) were not formally evaluated, the estimate of correlation (and $R$-squared value reported) should be interpreted cautiously.

Statistical methodology: While authors report an $R$ squared based on linear regression, no information on the regression model is provided. Details of the model used to generate the $R^{2}$ and $P$-value should be described. Is this based on regression model that only has BMI in the model? If there were other variables in the model, it should be stated what was included (additional variables also influence $R^{2}$ ). $R^{2}$ is probably not a clinically meaningful number. It tells you that for the particular model, a percent of the change is explained by the combination of factors in the model... and the rest is not explained by the model. $R^{2}$ is model dependent and there are number of other aspects of the model (and fit) that need to be considered.

Lack of comparators: Although the mean BMI of the patients is high (33), the evaluation was not exclusively done in obese or overweight patients (there are some patients with $B M I<30)$ and there isn't a comparison of change in lordosis among obese with non-obese patients, including those considered normal with respect to BMI. While BMI is a commonly used indicator of obesity, it measures total body mass and doesn't take into account lean muscle mass, which varies for men and women and with age. Factors other than obesity may affect lordosis, such as trunk length and ligamentous laxity (eg, Marfan's syndrome and Ehlers-Danlos patients). These potential confounding factors should be considered in further studies. To the extent that clinical factors may influence both BMI and change in lordosis, these factors should be measured and evaluated. 